\title{
Laryngeal Hamartoma in a Newborn
}

\author{
J SULTANA ${ }^{\mathrm{a}}$, NI NAZME ${ }^{\mathrm{b}}$, M SARKER ${ }^{\mathrm{c}}$, RB CHOWDHURY ${ }^{\mathrm{d}}$
}

Summary:

We report an interesting case of a neonate presenting immediately after birth with respiratory distress, inspiratory stridor and difficult intubation. During operative microlaryngoscopy, a well-delineated papilloma like elongated supraglottic mass was seen. The patient underwent surgery, and the diagnosis was confirmed histologically. Biopsy revealed tissue compatible with hamartoma including blood vessel, nerve bundle and cartilage plate. The mass was

\section{Introduction:}

Hamartomas are defined as a congenital malformation that consists of a focus of mature, locally derived tissue with abnormal histological architecture. Hamartomas are common among benign masses found in the lung. Head and neck manifestations are rare and can occur anywhere along the aerodigestive tract. Hamartomas affecting the larynx are extremely rare with less than twenty well-documented cases in the literature ${ }^{1}$. Hamartomas of the larynx are rare benign entities that can be locally destructive and cause airway obstruction. Recurrence rate is $20 \%$, often associated with incomplete removal ${ }^{2}$.

We report a case of supraglottic hamartoma causing airway obstruction in a newborn necessitating laryngoscopic removal of the mass. The laryngeal hamartoma should be removed in the newborn period to avoid tracheostomy, because decanulation after tracheostomy in infants can be difficult ${ }^{3}$.

a. Dr. Jesmin Sultana, Colonel, Child Specialist \& Neonatologist, Combined Military Hospital, Dhaka.

b. Dr. Nure Ishrat Nazme, Major, Child Specialist, Combined Military Hospital, Dhaka.

c. Dr. Mukta Sarker, Major, Child Specialist, Combined Military Hospital, Dhaka.

d. Dr. Rehana Begum Chowdhury, Brigadier General, Neonatologist, Head of the Dept. of Paediatrics, AFMC, Dhaka.

Address of Correspondence: Dr. Jesmin Sultana, Colonel, Child Specialist \& Neonatologist, Combined Military Hospital, Dhaka. E-mail: jesminpaed@yahoo.com

Received: 2 May, 2015

Accepted: 17 June, 2016 removed by direct laryngoscopy. Neonatal laryngeal hamartomas are extremely rare. In general, an excellent prognosis is associated with these lesions, and the treatment of choice is endoscopic surgical excision. To our knowledge, this is the first description of a neonatal laryngeal hamartoma in Bangladesh.

Keywords: Neonate, Larynx, Hamartoma

(J Bangladesh Coll Phys Surg 2016; 34: 172-174)

\section{Case report:}

The patient was a full term, female newborn who presented immediately after birth with inspiratory stridor and respiratory distress (Fig.-1). The baby cried 5 minutes after birth following resuscitation. She was on mechanical ventilator just after admission to Neonatal Intensive Care Unit due to respiratory difficulty and constant lower oxygen saturation. But intubation could be done with difficulty. The baby was delivered by Lower Uterine Cesarian Section (LUCS) at term due to placenta previa and history of antepartum hemorrhage. The baby was extubated after clinical improvement but again became lethargic and cyanosed with increasing intensity of stridor within a few hours. She remained event-free when endotracheal intubation was maintained with or without ventilator support. Each time intubation was very difficult. Serial chest radiographs demonstrated intermittent atelectasis and low lung volumes with hypoventilatory changes.

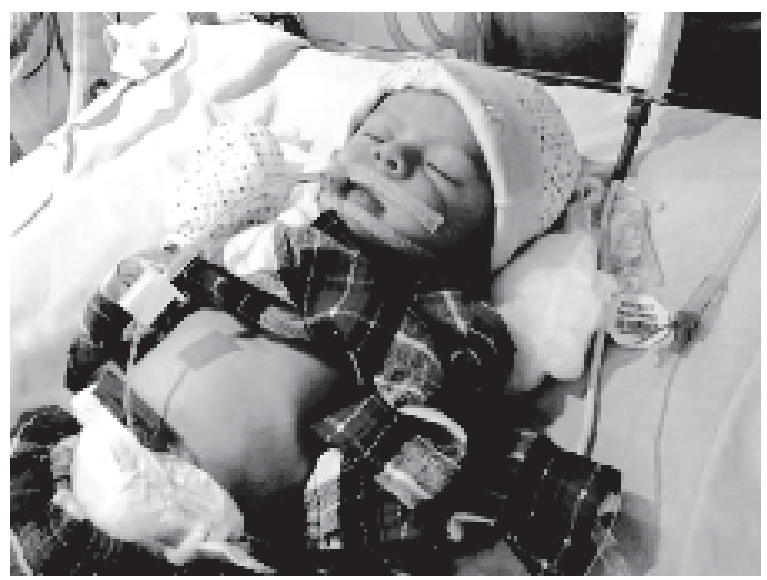

Fig.-1: Newborn with laryngeal hamartoma 
Then Otolaryngologist was consulted and flexible laryngoscopy at the bedside revealed a large, obstructing polypoidal mobile mass in supraglottis with suspicion of a supraglottic polyp. Operative microlaryngoscopy further characterized a well delineated, large papilloma like elongated supraglottic mass which was firm to hard in consistency. The mass was removed by direct laryngoscopy and airway was clear.

Histopathological examination confirmed a solid lesion with features of hamartoma including blood vessel, nerve bundle and cartilage plate. She was extubated on postoperative day 1 and the immediate postoperative course was uneventful. A trial of oral feeding was initiated on postoperative day 3, which resulted in increased work of breathing and concern for aspiration. Oral feedings were withheld, and the patient was brought back to the operating room for a follow up endoscopy where she was seen to have good healing. A nasogastric tube was placed for feeds.

Consequently, the baby was maintained on enteral feeds with plans for clinical re-evaluation. At a 2 month followup visit, the patient demonstrated no respiratory complaints or flexible fiberoptic laryngoscopy findings of recurrence. She is now 9 months old and doing well without any respiratory symptoms or feeding difficulties (Fig.-2).

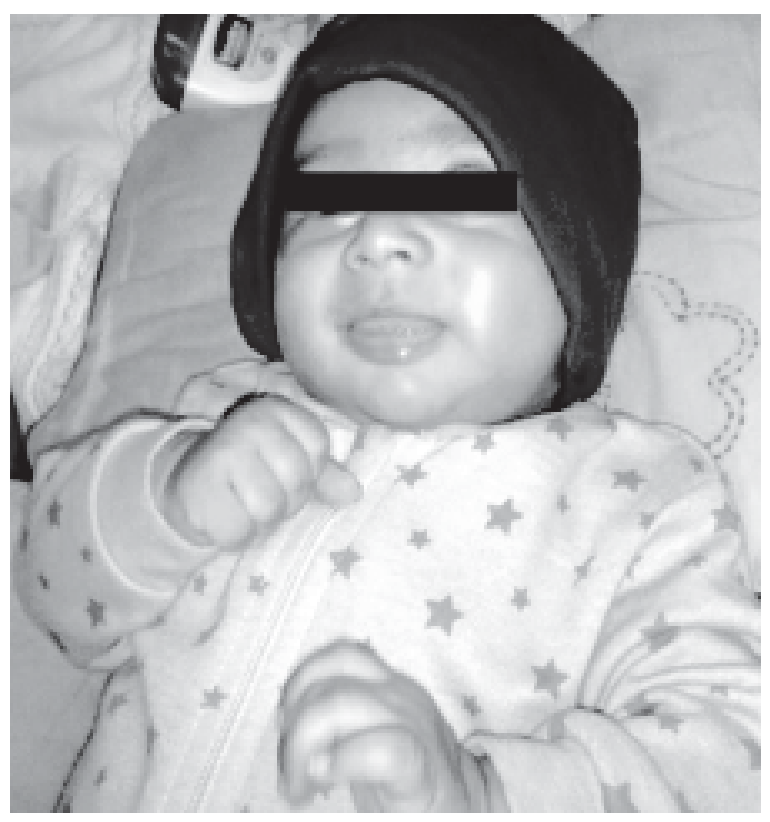

Fig.-2: The newborn infant with laryngeal hamartoma after recovery

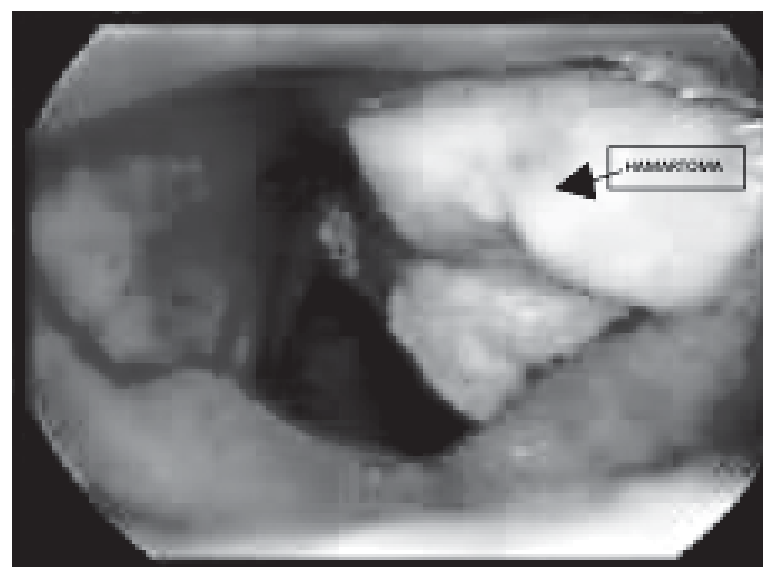

Fig.-3: Laryngoscopic view of laryngeal hamartoma

\section{Discussion:}

Hamartomas are simple and spontaneous growth composed exclusively of components derived from local tissue. The growth produces an excessive number of cells that reach maturity and then ceases to reproduce, so that the growth is self-limiting. Hamartomas are basically benign malformations although they often present many clinical features of a neoplasm, ${ }^{4}$.

We report an extremely rare case of a neonatal laryngeal hamartoma causing stridor and respiratory compromise since birth (Fig.-3). Linder describes a similar case of a neonatal boy presenting with respiratory distress secondary to a laryngeal hamartoma ${ }^{5}$. Windfuhr described a series of 10 pediatric patients with laryngeal hamartomas, presenting at a various ages, with an average age of 1.9 years. The most common presenting symptoms were stridor, dysphonia, and hoarseness, and most commonly affected the supraglottis ${ }^{1}$.

Another possible clinical presentation in laryngeal hamartoma is asphyxia described by Bouzouita in a case report. Our patient also presented with asphyxia. Asphyxia can occur according to the volume of the lesion, demanding prompt treatment as an emergency ${ }^{4}$. Some of these disorders were discovered during surgical procedures, when the lesion made intubation difficult ${ }^{5}$. In the case reported by us, the intubation was difficult drawing special attention to laryngeal pathology and considered consultation with Otolaryngologist and Anaesthesiologist. Our case is also similar to a case reported by Leoncini et al. however, they described a 3 month old infant who presented with progressive airway symptoms ${ }^{2}$. 
Clinically and histopathologically, our patient appears to have manifestations of the glandular hamartoma described by Sahhar and Leoncini et al ${ }^{2,6}$. In addition to the unique histopathology, diagnostic yield of crosssectional imaging with magnetic resonance imaging (MRI) is very crucial. . It may be advisable to use to evaluate the lesion's vascularity and potential involvement with other critical structures. But it has limited role in visualising neonatal laryn $x^{1}$. Again, microlaryngocopy is the primary modality for diagnosis of these lesions; the operative goal should be to primarily focus on maintaining function, as these lesions generally have an excellent prognosis ${ }^{7}$.

The pathophysiologic mechanism underlying the origin of oropharyngeal polyp is unknown. Polypoid lesions may have many different histologic abnormalities. Therefore, it is important to consider other neoplastic and non-neoplastic conditions that may present as a polyp in the differential diagnosis. These include hamartomas, inflammatory polyps, lipomas, hemangiomas, lymphangiomas, schwannomas, and other unusual neoplasms, such as carcinoid tumors and chemodectomas $^{8}$. Histopathological examination of the resected polyp in our case revealed features of hamartoma including blood vessel, nerve bundle and cartilage plate.

In present case, the polypoidal mass was removed by direct laryngoscopy with excellent prognosis in subsequent follow up. The lesion was treated in a similar fashion, with an excellent functional result and prognosis described by Leoncini ${ }^{2}$.The airway in another case was secured via tracheostomy, and the lesion was resected endoscopically with a carbon dioxide laser. A subtotal resection was achieved, but functionally, the patient did well and was eventually decannulated at 33 months of age ${ }^{5}$. The lesions were fatal in two neonates in another report, but generally, an excellent prognosis can be expected with complete surgical removal as the treatment of choice. In addition, while benign, there was report of one case with histologic change observed at recurrence ${ }^{1}$. In general, an excellent prognosis is associated with these lesions, and the treatment of choice is endoscopic surgical excision with complete recovery. ${ }^{9}$

\section{Conclusion:}

Neonatal laryngeal hamartoma is extremely rare which is primarily a benign disease with excellent prognosis after complete endoscopic removal. The aim of our report is to underline consideration of laryngeal hamartoma by paediatricians as an important cause of airway obstruction in newborn with difficult intubation.

\section{Referances:}

1. Windfuhr JP. Laryngeal hamartoma. Acta OtoLaryngologica 2004;124:301-308.

2. Leoncini G, Maio V, Mirabile L, Baggi R, Franchi A. Glandular hamartoma of the larynx: report of a case. Auris Nasus, Larynx 2008; 35:149-151.

3. Jakubýìková J, Haruštiak S, Galbavý Š, Chebenová J. Laryngeal hamartoma: surgical management. M. Clin Pathol 2006; 59(5):542-545.

4. Bouzouita K, Sriha B, Mani R, Bouzouita H. An unusual cause of neonatal respiratory distress: nasopharyngeal hamartoma, 2 cases report. Rev Laryngol Otol Rhinol (Bord) 2001; 122(3):171-3.

5. Linder H. Hamartoma of the larynx causing neonatal respiratory distress. J Laryngol Otol 1997; 111:166-168.

6. Sahhar HS, Marra S, Boyd C, Akhter J. Ectopic subglottic thymic cyst: a rare cause of congenital stridor. Ear Nose Throat J 2003; 82:873-874.

7. Carpenter LM, Merten DF. Radiographic manifestations of congenital anomalies affecting the airway. Radiologic Clinics of North America 1991; 29:219-240.

8. Borges A, Bikhazi H, Jeffrey P, Wensel. Giant Fibrovascular Polyp of the Oropharynx: Case Report. Am J Neuroradiol 1999; 20:1979-1982.

9. Cheng J, McKay E, Rizzi MD. Congenital hamartoma with ectopic thymus causing airway compromise in a neonate. International Journal of Pediatric Otorhinolaryngology Extra 2013; 8(1):13-15. 